\title{
COVID-19 outbreak in children with cancer in a tertiary care center of a developing country
}

\author{
Abdul Mํㄹ Prasanth Siri ${ }^{1}$, Debasish Sahoo ${ }^{1}$, Jagdish Meena ${ }^{1}$, Aditya Gupta ${ }^{1}$, and Rachna \\ Seth $^{1}$ \\ ${ }^{1}$ All India Institute of Medical Sciences
}

October 16, 2020

\begin{abstract}
Data on the prevalence and outcomes of coronavirus disease (COVID-19) in children with cancer is limited, more so from developing countries. We aimed to evaluate the prevalence, severity and outcome of COVID-19 in pediatric oncology patients by implementing a screening and testing plan. Among 104 children tested, the rate of COVID-19 positivity was $15 \%$ and $3 \%$ in symptomatic and asymptomatic respectively. Majority had mild illness with uneventful recovery, whereas neutropenia increased the risk of serious illness. Prolonged virus shedding with a median time to negativity of 21 days was observed.
\end{abstract}

\section{Introduction}

The outbreak of severe acute respiratory syndrome coronavirus 2(SARS-CoV-2) causing the coronavirus disease(COVID-19) pandemic in 2020 started at the beginning of December 2019 in Wuhan, China, and then spread to many countries around the world. It has been observed that elderly people and patients with immune-deficits are at high risk of mortality from the infection.Children, in contrast to adults, are not commonly affected by the infection and, if infected, usually are less symptomatic ${ }^{1}$ Patients on anticancer therapy may be at a higher risk of developing infectious complications due to their greater level of immunosuppression. Data on the prevalence of SARS-CoV-2 infection in children with cancer is limited, more so from developing countries. To evaluate the prevalence and the severity of SARS-CoV- 2 in pediatric oncology patients, we instituted a screening and testing plan in patients admitted to our unit, which is a tertiary referral care centre in North India.

\section{Methods}

Children with cancer and their guardians attending pediatric oncology services (day care/in house) between May 12 and July 31were screened and testing was carried out in 3 cohorts of patients 1) Patients who showed definite symptoms of COVID-19 infection like fever, cough, shortness of breath, sore throat, myalgia (symptom positive) 2) Those who had contact with COVID-19 positive patients and asymptomatic(screen positive) and 3) Asymptomatic patients prior to inpatient admission or invasive/aerosol generating procedures like biopsy/fine needle aspiration (screen and symptom negative). Testing was done using qualitative reverse transcriptase polymerase chain reaction (RT-PCR). Patients were followed up and data were gathered prospectively.

\section{Results}

Between May 12 and July 31, a total of 104 tests for COVID-19 were done in pediatric oncology patients. Sixty-eight patients were symptom positive, $\mathbf{5}$ patients were screen positive and $\mathbf{3 1}$ patients were screen and symptom negative. Among the total patients screened and tested, SARS- CoV-2 was positive in 11 patients $(\mathbf{1 0 . 5 \%})$ out of which10 were in the first cohort (15\%), 1 in the second cohort and 
none in third cohort. (Table 1) . Common symptom was shortness of breath ( $\mathrm{n}=5$ patients, $45 \%$ ), fever $(\mathrm{n}=5,45 \%)$, body-aches $(\mathrm{n}=3,27 \%)$, neurological symptoms $(\mathrm{n}=2,18 \%)$ and cough/coryza $(\mathrm{n}=2,18 \%)$. Chest X-ray abnormalities were found in 4 patients.

Of the 104 patients ( 82 male and 22 female) tested, 11 (10\%)had positive test results. The rate of COVID19 positivity among asymptomatic patients was $\mathbf{3} \%(1 / 36)$. Male : female ratio was $2.7: 1$ among positive patients. All patients except three (one patient was lost to follow up) required admission for variable length of time. Three patients required ICU care out of which 2 succumbed to their illness. Both patients who died were recently diagnosed as acute lymphoblastic leukemia and had coexisting neutropenia, septicemia and neurological involvement. Third patient had pneumonia and febrile neutropenia recovered uneventfully. All other patients had mild symptoms and were advised home quarantine after an initial period of hospital admission. Repeat COVID testing could be done in 8 patients. Median time to negativity of COVID-19 by RT-PCR was 21 days (IQR:10 - 27.7). Characteristics of COVID positive patients are shown in

Table 2.

\section{Discussion}

Spectrum of COVID -19 infection in children include asymptomatic infections, fever, cough, sore throat, myalgia, shortness of breath, abdominal pain and diarrhoea. Rarely it manifests as a systemic disease leading to myocarditis, meningoencephalitis, Kawasaki disease like illness and macrophage activation syndrome. Literature shows that COVID-19 infection takes a milder course in children with better prognosis compared to adults. ${ }^{1}$ The reason for the difference is not clearly understood, but is hypothesized due to relative paucity of angiotensin-converting enzyme (ACE-2) cell receptors present on the respiratory mucosa of children, where SARS-CoV-2 S protein binds. Trained immunity due to live vaccines and frequent viral infections in childhood along with good lung regenerative capacity also contributes to less severe infection in children. ${ }^{2}$ In a large review of COVID - 19 in children, more than $90 \%$ of the children were asymptomatic or had mild to moderate disease, $5.2 \%$ had severe disease and $0.6 \%$ had critical disease. ${ }^{3}$ More severe disease and prolonged viral shedding ( $>21$ days) can be caused by other human coronaviruses in immunocompromised patients. Cancer patients may behave differently as they are immunocompromised, frequently have associated febrile neutropenia and other co-morbidities due to chemotherapy or radiation. Polymicrobial infections are also common in neutropenic patients.

The first report of COVID-19 infection in a cancer child came from China when an 8-year-old child undergoing chemotherapy for T- cell ALL in Wuhan hospital developed respiratory failure, eventually requiring mechanical ventilation. ${ }^{4}$ Hrusak et al,subsequently published a flash survey on COVID-19 incidence and severity among children on anticancer treatment using a web-based form from 89 pediatric hematology/oncology units over 32 countries. Over 200 children were tested out of which 9 was positive for COVID-19 infection. Co existent febrile neutropenia was found in 5 of these positive patients. Eight of the nine cases had asymptomatic to mild disease, and recovered uneventfully. ${ }^{5}$ Researchers from Memorial Sloan Kettering Cancer Centre (MSK) retrospectively analysed 178 paediatric cancer patients who were tested for COVID-19 and found that $11 \%$ had positive test results. The rate of positivity was $29 \%$ among symptomatic patients and 2.5\% among asymptomatic patients. They also found incidence of COVID-19 was significantly more in males. ${ }^{6}$ In another report from France, 5 children with cancer had proven COVID-19 infection including two hematopoietic transplant recipients, data on their follow up was not available. ${ }^{7}$

Although our prospective analysis showed that majority of the children had mild symptoms and recovered completely, of note there was 2 mortality. Both of them had concurrent febrile neutropenia. Case 1 (BALL) developed features of meningoencephalitis with raised intracranial tension and E. Coli septicemia. It is highly likely that the cause of death may be due to febrile neutropenia with polymicrobial sepsis and multiorgan dysfunction than directly attributing to COVID-19 alone. Case 6 (B-ALL) was improving initially, subsequently deteriorated with fever, hypoxia, respiratory distress and status epilepticus. Intracranial hemorrhage due to severe thrombocytopenia as a possible cause of death could not be ruled out because 
neuroimaging could not be done. Meningoencephalitis as a manifestation of SARS-CoV-2 is also well described in literature. ${ }^{8}$ It is also worth mentioning that all the care takers of infected children, except one were negative for COVID-19, which support the hypothesis that children are less likely to spread disease. Another important consideration is the duration of viral shedding which had a great impact on the timely resumption of chemotherapy. In our cohort of patients, we found that the median period for the PCR test to become negative from respiratory samples was 21 days. This is slightly high when compared with the previous published data in which one study found the median duration of viral shedding to be $\mathbf{1 5}$ days and was more in symptomatic patients compared to asymptomatic whereas; in another study the mean duration of virus shedding ranged from $\mathbf{1 1} \pm \mathbf{5}$ days. ${ }^{9,10}$

In spite of being a tertiary referral cancer centre, we were able to curb the spread of infection to a good extent by implementing some specific hospital admission protocols. These include routine screening and testing of all symptomatic and asymptomatic patients prior to admission, limiting the number of care givers inside pediatric ward to one, frequent hand washing, compulsory face mask use by child and care givers, use of personal protective equipments by health staffs, social distancing, staggering appointments for chemotherapy and procedures and utilizing teleconsultation for follow up of patients. A triage system was also introduced for our patients dividing them into very low risk, low risk, intermediate risk and high risk that helped the team to make decisions. Only high risk patients were administered chemotherapy from day-care/inhouse admission as delay in treatment was likely to affect outcome adversely in this group. ${ }^{11,12}$

\section{Conclusion}

Although our analysis is limited by small number of patients, the data show that COVID-19 infected cancer patients may be at increased risk for mortality especially if they have associated febrile neutropenia. Majority have mild illness with uneventful recovery. We also found that the rate of positivity among asymptomatic patients was very low. Strict adherence to preventive strategies and development of institutionalised protocols is mandatory to manage this pandemic. More data is required in pediatric cancer patients to clearly understand the clinical behaviour and trajectory of the COVID-19 illness.

\section{Conflict of interests}

None declared by the authors

\section{Acknowledgements}

Nil

\section{References}

1. Ludvigsson JF. Systematic review of COVID-19 in children shows milder cases and a better prognosis than adults. Acta Paediatr. 2020.

2. Nitin Dhochak, Tanu Singhal, S. K. Kabra, et al. Pathophysiology of COVID-19: Why Children Fare Better than Adults? The Indian Journal of Pediatrics (July 2020) 87(7):537-546.

3. Dong Y, Mo X, Hu Y, et al. Epidemiological characteristics of 2143 pediatric patients with 2019 coronavirus disease in China. Pediatrics. 2020

4. Chen Z, Xiong H, Li JX, et al. COVID-19 with post-chemotherapy agranulocytosis in childhood acute leukemia: a case report. Zhonghua Xue Ye Xue Za Zhi. 2020;41:E004.

5. Hrusak O, Kalina T, Wolf J, et al. Flash survey on severe acute respiratory syndrome coronavirus-2 infections in paediatric patients on anticancer treatment. Eur J Cancer . 2020;132:11-16.

6. Farid Boulad, Mini Kamboj, Nancy Bouvier, et al. COVID-19 in Children With Cancer in New York City. JAMA Oncology, May 13,2020.

7. Nicolas Andre, Jeremie Rouger-Gaudichon, Benoit Brethon, et al. COVID-19 in pediatric oncology from French pediatric oncology and hematology centers: High risk of severe forms? PediatrBloodCancer. $2020 ; \mathrm{e} 28392$. 
8. Mark A Ellul, Laura Benjamin, Bhagteshwar Singh, et al. Neurological associations of COVID-19. Lancet Neurol July 2020.

9. Yingying Lu, Yi Li, Wenyue Deng, et al. Symptomatic Infection is Associated with Prolonged Duration of Viral Shedding in Mild Coronavirus Disease 2019. Pediatr Infect Dis J 2020;39:e95-e99.

10. Xu, Cecilia L. H; Raval, Manjri; Schnall, Jesse A; et al. Duration of Respiratory and Gastrointestinal Viral Shedding in Children With SARS-CoV-2. The Pediatric Infectious Disease Journal: June 30, 2020 .

11. Bouffet E, Challinor J, Sullivan M, et al. Early advice on managing children with cancer during the COVID-19 pandemic and a call for sharing experiences. Pediatr Blood Cancer . 2020;2:e28327.

12. Seth R, Das G, Prasanth S, et al. Delivering pediatric oncology services during a COVID-19 pandemic in India. Pediatr Blood Cancer. 2020;e28519.

\section{Hosted file}

Table 1 PBC.pdf available at https://authorea.com/users/329710/articles/487031-covid-19outbreak-in-children-with-cancer-in-a-tertiary-care-center-of-a-developing-country

\section{Hosted file}

Table 2 PBC.pdf available at https://authorea.com/users/329710/articles/487031-covid-19outbreak-in-children-with-cancer-in-a-tertiary-care-center-of-a-developing-country 